\title{
High-density linkage map around the root aerenchyma locus Qaer1.06 in the backcross populations of maize Mi29× teosinte "Zea nicaraguensis"
}

\author{
Yoshiro Mano* and Fumie Omori \\ Forage Crop Breeding Research Team, National Institute of Livestock and Grassland Science, 768 Senbonmatsu, Nasushiobara, Tochigi \\ 329-2793, Japan
}

The teosinte Zea nicaraguensis forms constitutive aerenchyma in the root cortex as observed in flooding tolerant wetland plants. We have previously identified a quantitative trait locus (QTL) controlling aerenchyma formation under non-flooding conditions on chromosome 1 (Qaer1.06) using 214 individuals of a maize Mi29 $\times$ Z . nicaraguensis $\mathrm{BC}_{2} \mathrm{~F}_{1}$ population. The present studies objective was to increase the marker density around the Qaer1.06 locus, which is essential for the development of near-isogenic lines possessing the aerenchyma-forming gene with only a small region of the Z. nicaraguensis chromosome segment. A survey of $62 \mathrm{SSR}$ and 38 insertion/deletion (INDEL) markers identified a total of 36 useful markers in the region of interest. These were selected for the construction of a linkage map in a 214 individual $\mathrm{BC}_{2} \mathrm{~F}_{1}$ and a 123 individual $\mathrm{BC}_{4} \mathrm{~F}_{1}$ population from a cross between Mi29 $\times$ Z. nicaraguensis. Using the $\mathrm{BC}_{4} \mathrm{~F}_{1}$ population, we performed QTL mapping and the results indicated that a QTL for aerenchyma formation under non-flooding condition was located on chromosome 1 (bin 1.05) at a position that is near to Qaer1.06. The markers obtained here should be useful for the development of high quality near-isogenic lines possessing the Qaer1.05-6.

Key Words: aerenchyma, flooding, near-isogenic line, maize, quantitative trait locus, teosinte.

\section{Introduction}

Aerenchymatous root in plants perform an important role for adaptation in oxygen- deficient flooded or waterlogged soils because oxygen is provided to the root tip by diffusion through the aerenchyma. Maize (Zea mays L. ssp. mays) forms lysigenous aerenchyma in the cortical cells of roots following hypoxia or low oxygen levels during flooding, and physiological and molecular mechanisms associated to this character have been widely investigated (Jackson et al. 1985a, He et al. 1996, Saab and Sachs 1996, Gunawardena et al. 2001, Subbaiah and Sachs 2003). This floodinginducible aerenchyma formation is also observed in upland crops such as barley (Pang et al. 2004), wheat (Huang et al. 1994, Watkin et al. 1998) and triticale (Watkin et al. 1998), and several reports suggest that flooding tolerant varieties possess larger inducible aerenchyma compared to intolerant varieties (Boru et al. 2003, Setter and Waters 2003, Pang et al. 2004, Zaidi et al. 2007). From an evaluation of over 4,000 accessions, Takeda (1989) has identified and selected barley genotypes that exhibit a superior tolerance to flooded conditions and the tolerant genotypes abundantly developed aerenchymatous adventitious roots (Stanca et al. 2003).

The teosintes represent a wild relative of maize typically found in regions of Mexico, Guatemala, Honduras and

Communicated by T. Komatsuda

Received October 6, 2009. Accepted November 6, 2009.

*Corresponding author (e-mail: mano@affrc.go.jp)
Nicaragua that receive frequent levels of precipitation could be a valuable germplasm resource for developing flooding tolerant maize (Bird 2000, Iltis and Benz 2000, Mano and Omori 2007). The study of the teosintes also will enhance the understanding of the mechanism of aerenchyma formation because hybridization between non aerenchymaforming maize and aerenchyma-forming teosinte are easily generated.

Among the several teosintes classified by Doebley and Iltis (1980), Iltis and Doebley (1980) and Iltis and Benz (2000), constitutive aerenchyma that develop in the absence of hypoxia or low oxygen is expressed in Z. luxurians and $Z$. nicaraguensis in well-aerated greenhouse environments (Ray et al. 1999, Mano et al. 2006) and in upland field conditions (Y. Mano, unpublished). A similar situation is known with flooding tolerant rice (Jackson et al. 1985b, Colmer 2003) and other wetland species (Smirnoff and Crawford 1983, Justin and Armstrong 1987). Constitutive aerenchyma is typically in these species; however, during flooding conditions, the degree of aerenchyma in rice and teosinte is increased and additional complete (functional) aerenchyma channels can be developed more rapidly compared to inducible aerenchyma-forming upland plants. Therefore, constitutive aerenchyma in plants is an advantageous consequence for adaptation to temporary flooding conditions (Colmer and Voesenek 2009).

In prior studies, we have focused on the presence of constitutive aerenchyma formation in teosinte Z. nicaraguensis. In quantitative trait locus (QTL) analyses to determine the 
capacity of constitutive aerenchyma formation, using teosintes $Z$. nicaraguensis and Z. luxurians, we have recently identified QTLs associated to aerenchyma formation (Mano et al. 2007, 2008). Therefore, the transfer of aerenchyma formation gene(s) from teosinte to maize can be achieved via molecular marker-assisted backcrossing program. Once the experimental material (e.g. isogenic line) is obtained, transcriptome analyses will be undertaken to reveal the mechanism of aerenchyma formation. This approach has been applied to identify candidate gene associated with maize root responses towards environmental stimuli (Hochholdinger and Tuberosa 2009).

In a previous study using a $214 \mathrm{BC}_{2} \mathrm{~F}_{1}$ individual population of maize Mi29 $\times$ Z. nicaraguensis, a QTL for aerenchyma formation under non-flooding conditions was located to chromosome 1 (bin 1.06) and designated as Qaer1.06. The objectives of this study were (1) to increase molecular markers around the aerenchyma locus Qaer1.06; (2) to verify an effect of the Qaer1.06 in the genetic background of maize Mi29; and (3) to search for an additional QTL for aerenchyma formation other than the Qaer1.06. These results will be essential to determine a precise position of the Qaer1.06, develop high-quality near-isogenic line possessing the Qaer1.06 useful for gene isolation as well as the development of flooding tolerant maize by marker-assisted selection.

\section{Materials and Methods}

\section{Plant materials}

Maize inbred Mi29, a line that does not form constitutive aerenchyma, and the teosinte $Z$. nicaraguensis (CIMMYT 13451) were crossed to form the initial $F_{1}$ hybrid and populations. Because Z. nicaraguensis is an outcrossing species, it was self-pollinated twice prior to crossing to Mi29, in order to form a more stable aerenchyma-forming S2 generation for use in the genetic analysis.

For the marker survey and construction of a high-density linkage map, an Mi29 $\times$ Z. nicaraguensis 214 individual $\mathrm{BC}_{2} \mathrm{~F}_{1}$ population (Mano and Omori 2008) was used for the analysis. A single $F_{1}$ plant derived from a cross between Mi29 $\times$ Z. nicaraguensis was backcrossed to Mi29 and 42 $\mathrm{BC}_{1} \mathrm{~F}_{1}$ plants were obtained. These $42 \mathrm{BC}_{1} \mathrm{~F}_{1}$ plants were backcrossed using $\mathrm{Mi} 29$ as the pollen parent to produce $\mathrm{BC}_{2} \mathrm{~F}_{1}$ seeds. $214 \mathrm{BC}_{2} \mathrm{~F}_{1}$ individuals grown from $4-6 \mathrm{BC}_{2} \mathrm{~F}_{1}$ seeds from each of the $42 \mathrm{BC}_{2} \mathrm{~F}_{1}$ spikes were developed.

To generate the $\mathrm{BC}_{4} \mathrm{~F}_{1}$ population, the same $\mathrm{F}_{1}$ plant that was used for the development of the $214 \mathrm{BC}_{2} \mathrm{~F}_{1}$ population, was crossed to Mi29. A single $\mathrm{BC}_{1} \mathrm{~F}_{1}$ plant was backcrossed to $\mathrm{Mi} 29$ three times to produce the $\mathrm{BC}_{4} \mathrm{~F}_{1}$ seeds. During the backcross process, SSR markers bnlg1832 (bin1.05), umc1128 (1.07) and their flanking markers that bracket 45 $\mathrm{cM}$ around the Qaer1.06 region were used in the selection process. Figure 1 shows the graphical genotype of a $\mathrm{BC}_{3} \mathrm{~F}_{1}$ plant used to develop the $\mathrm{BC}_{4} \mathrm{~F}_{1}$ mapping population. The frequency of heterozygous genotypes (Mi29/Z. nicaraguensis; shadowed regions) was 0.225 (data not shown), which is

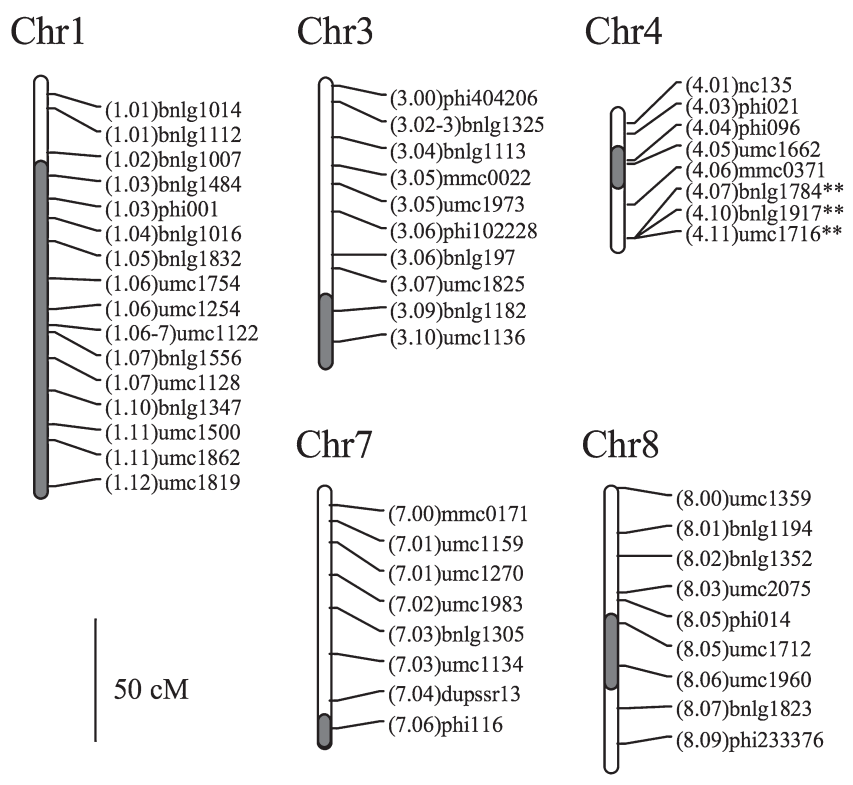

Fig. 1. Graphical genotype of a single $\mathrm{BC}_{3} \mathrm{~F}_{1}$ plant used to develop the $\mathrm{BC}_{4} \mathrm{~F}_{1}$ mapping population of the cross between Mi2 $9 \times$ Z. nicaraguensis. Shadowed regions indicate the fragments of heterozygous genotypes (Mi29/Z. nicaraguensis). Map distance is based on the study of Mano and Omori (2008).

higher compared to the expected value of 0.125 in the $\mathrm{BC}_{3} \mathrm{~F}_{1}$ generation.

\section{Root anatomy}

A total of $123 \mathrm{BC}_{4} \mathrm{~F}_{1}$ individuals were grown in a greenhouse maintained at a temperature of $30^{\circ} \mathrm{C}$ day $/ 25^{\circ} \mathrm{C}$ night with natural light at 13-14 hours day length. The degree of constitutive aerenchyma in the root cortex of six-leaf stage seedlings that grew under non-flooding condition was visually scored: 0 (no aerenchyma), 0.5 (partial formation), 1 (radial formation) and 2 (radial formation extended toward epidermis) as described in Mano et al. (2006). In addition, because the variation for the trait in the backcrossed population was not large, we used a more discrete scoring rating (i.e. $0.25,0.75$ and 1.5 ) to generate more accurate trait evaluation data. For the QTL analysis, we averaged evaluation scores at 10 and $15 \mathrm{~cm}$ from the root tips of two adventitious roots per individual for a total of 4 positions per individual.

\section{Survey of useful markers around the Qaer1.06}

Approximately $1-4 \mu \mathrm{g}$ of DNA was isolated from $50 \mathrm{mg}$ of fresh leaf tissue using the method described by Komatsuda et al. (1998). Based on maize GDB (http://www.maizegdb. org/), we selected 62 SSR markers and 38 insertion/deletion (INDEL) markers in the vicinity of the Qaer1.06 region from bin 1.05 to 1.07 .

To detect polymorphisms in the SSR and INDEL markers, $48 \mathrm{BC}_{2} \mathrm{~F}_{1}$ individuals were tested for each primer pair because the $Z$. nicaraguensis used in this experiment was not a pure line and amplicon size could differ within Z. nicaraguensis. The SSR analysis was performed as 
described by Mano et al. (2005).

For the INDEL marker analysis, all the forward primers were 5-end labeled with fluorescence dye FAM or HEX. Reaction mixtures contained $20 \mathrm{ng}$ of genomic DNA, $0.25 \mu \mathrm{M}$ of fluorescently labeled forward and unlabeled reverse primers, $200 \mu \mathrm{M}$ dNTPs, $3.5 \mathrm{mM} \mathrm{MgCl}_{2}$ (final concentration), 0.25 units of Taq DNA polymerase (Qiagen $\mathrm{GmbH}$, Hilden, Germany) and its corresponding reaction buffer with Qsolution for a total volume of $10 \mu \mathrm{L}$. The amplification conditions described in the MaizeGDB were followed: $3 \mathrm{~min}$ at $94^{\circ} \mathrm{C}$ followed by 30 cycles of $30 \mathrm{~s}$ at $94^{\circ} \mathrm{C}, 45 \mathrm{~s}$ at $60^{\circ} \mathrm{C}$ and $90 \mathrm{~s}$ at $72^{\circ} \mathrm{C}$, and a final extension at $72^{\circ} \mathrm{C}$ for $10 \mathrm{~min}$. PCR products were separated on a $20 \mathrm{~cm}, 5 \%$ denaturing polyacrylamide gel at $360 \mathrm{~V}$ for $1 \mathrm{~h} 15 \mathrm{~min}$ and then scanned in the Molecular Imager (Bio Rad, Hercules, CA).

\section{Map construction}

In the $\mathrm{BC}_{2} \mathrm{~F}_{1}$ population, markers were grouped according to a two-point analysis with $\mathrm{LOD}>3.0$ with a recombination fraction of 0.25 . The order of the markers was determined by the "three-point" and "order" command, first at LOD > 3.0, then at LOD > 2.0 using MAPMAKER/EXP 3.0 (Lander et al. 1987). Map distance (Haldane units) was computed in the $\mathrm{BC}_{2} \mathrm{~F}_{1}$ model using the QGene program (Nelson 1997). In the $\mathrm{BC}_{4} \mathrm{~F}_{1}$ population, the same procedure was applied for the construction of a linkage map with the single exception that the map distance was computed in the $\mathrm{BC}_{1} \mathrm{~F}_{1}$ model using the MAPMAKER/EXP 3.0.

\section{QTL analysis}

In the $\mathrm{BC}_{4} \mathrm{~F}_{1}$ population, composite interval mapping (CIM) was applied to map the QTLs controlling aerenchyma formation in the $\mathrm{BC}_{1} \mathrm{~F}_{1}$ model using the software package Windows QTL Cartographer Version 2.5 (Wang et al. 2006). CIM was run with $2 \mathrm{cM}$ walk speed applying the default parameters (model 6; 5 for control markers, $10 \mathrm{cM}$ for window size and forward regression method) in the program. The experiment-wise significance threshold level was defined as the 50th highest LOD value of 2.0 by running 1,000 permutations (Churchill and Doerge 1994), corresponding to an experiment-wise Type-I error rate of 0.05 .

\section{Results}

\section{Aerenchyma formation}

The average aerenchyma scores were $0.0 \pm 0.0$ (mean \pm standard deviation) for Mi29 $(\mathrm{n}=10)$ and $2.0 \pm 0.1$ for Z. nicaraguensis $(\mathrm{n}=10)$ (Fig. 2). Scores of the $\mathrm{BC}_{4} \mathrm{~F}_{1}$ individuals had a continuous distribution (average $0.2 \pm 0.2$, Fig. 3) and a segregant with high degree of aerenchyma that was equivalent to $Z$. nicaraguensis was not observed (Fig. 2C and Fig. 2D).

\section{Survey of useful markers around the Qaer1.06}

Of the 62 SSR tested, 24 (38.7\%) showed clear polymorphism between Mi29 and Z. nicaraguensis. Another 19

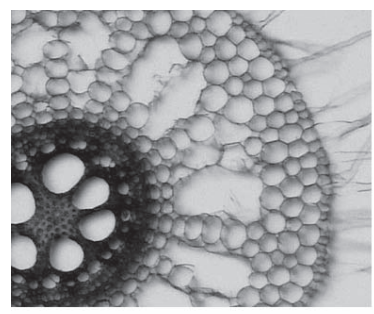

(A) Z. nicaraguensis

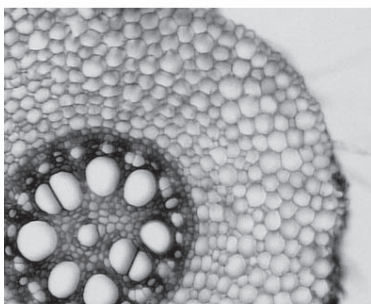

(B) Mi29

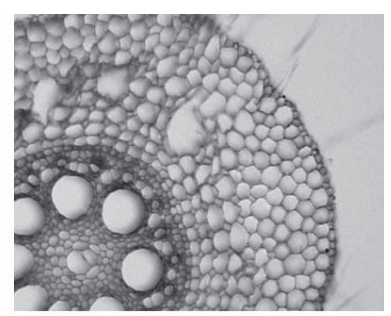

(C) $\mathrm{BC}_{4} \mathrm{~F}_{1} \# 10$

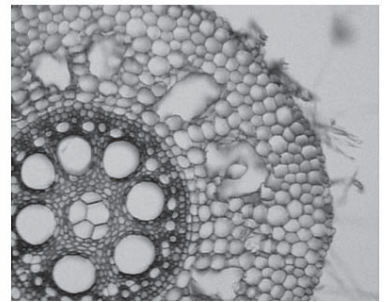

(D) $\mathrm{BC}_{4} \mathrm{~F}_{1} \# 16$
Fig. 2. Cross sections of adventitious roots at $10-15 \mathrm{~cm}$ from the root tip that emerged from the second node in six-leaf stage seedlings in non-flooding condition. Presence of aerenchyma is shown in (A) Z. nicaraguensis (score 2), (C) $\mathrm{BC}_{4} \mathrm{~F}_{1} \# 10$ (score 0.5) and (D) $\mathrm{BC}_{4} \mathrm{~F}_{1}$ \#16 (score 1) and the lack of aerenchyma in (B) Mi29 (score 0).

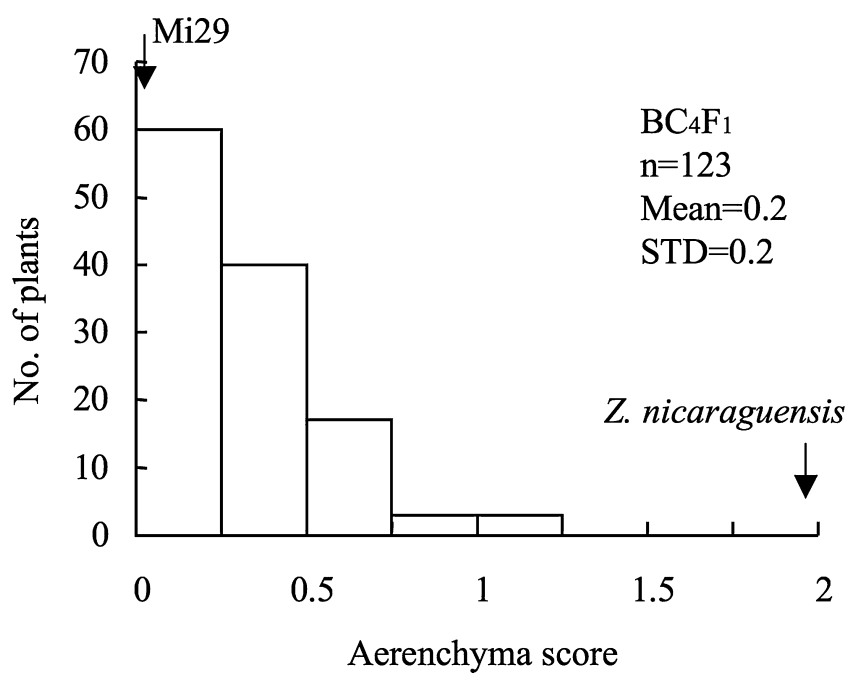

Fig. 3. Frequency distribution for aerenchyma formation in the Mi29 $\times Z$. nicaraguensis $\mathrm{BC}_{4} \mathrm{~F}_{1}$ population. Average score of two adventitious roots at $10 \mathrm{~cm}$ and $15 \mathrm{~cm}$ from the root tip (four positions in total) were used to determine the value for each $\mathrm{BC}_{4} \mathrm{~F}_{1}$ individual.

(30.6\%) showed only a monomorphic fragment and the remaining 19 (30.6\%) gave no or only weak amplifications. For the 38 INDEL marker analyses, 12 (31.6\%) showed clear polymorphism between the parents, $14(36.8 \%)$ showed only a monomorphic fragment and $12(31.6 \%)$ did not show any clear amplifications. In the marker survey, a total of 36 useful markers (24 SSRs and 12 INDELs) were identified and utilized for further mapping analyses.

\section{Map construction}

We constructed a linkage map of the 214 individual Mi29 


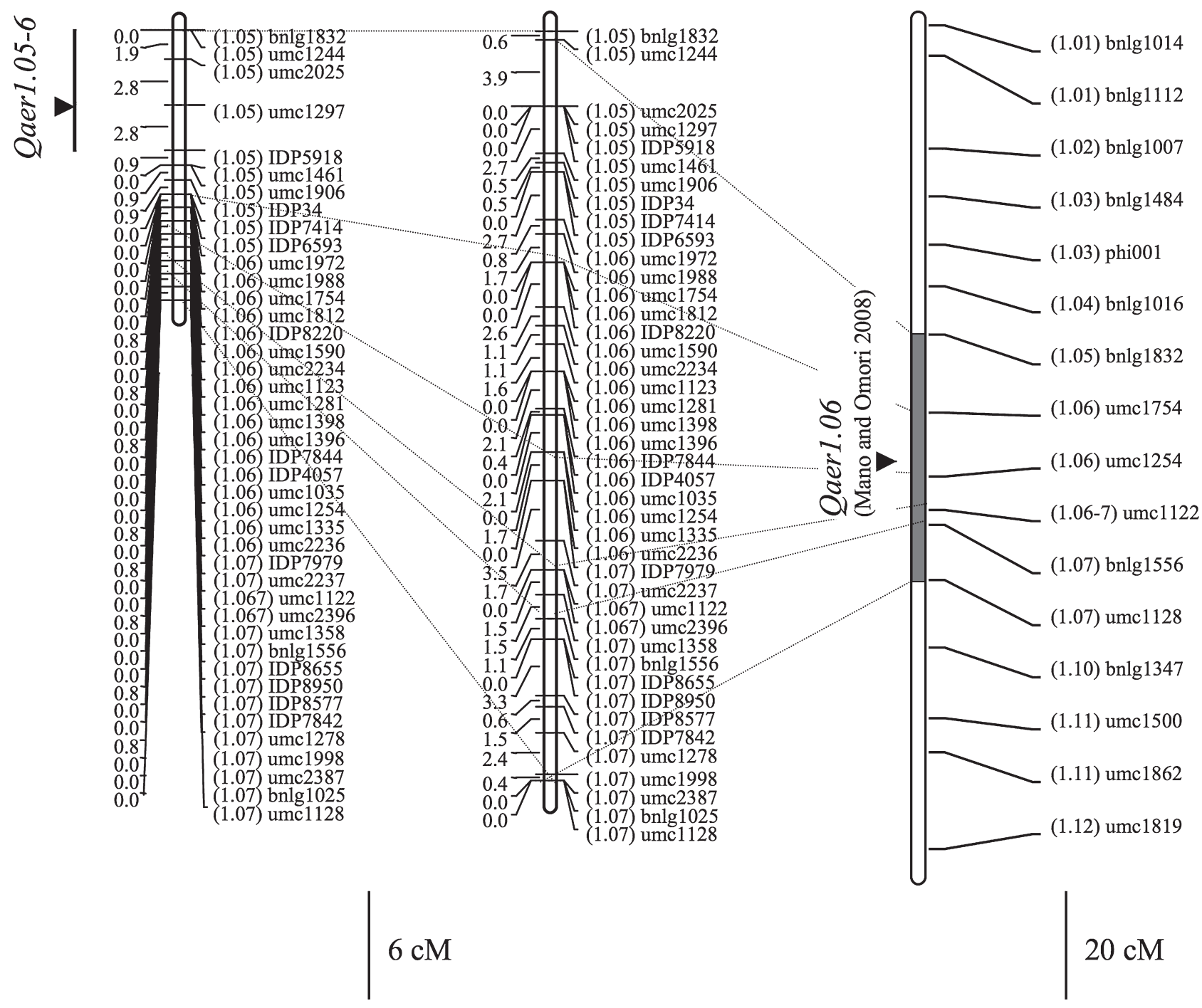

(A) $\mathrm{BC}_{4} \mathrm{~F}_{1}$

(B) $\mathrm{BC}_{2} \mathrm{~F}_{1}$

(C) $\mathrm{BC}_{2} \mathrm{~F}_{1}$

(Mano and Omori 2008)

Fig. 4. Linkage map of the $\mathrm{BC}_{4} \mathrm{~F}_{1}$ (A), $\mathrm{BC}_{2} \mathrm{~F}_{1}$ (B; this study) and $\mathrm{BC}_{2} \mathrm{~F}_{1}(\mathrm{C}$; Mano and Omori 2008) populations of the cross between Mi29 $\times$ $Z$. nicaraguensis. Short arms of the chromosome are on top. The scale is in centimorgans (Haldane units). Closed arrowheads indicate the position of the peak LOD. Bin numbers, traditional methods for referring to the location/position of markers, are in parentheses before marker names.

$\times Z$. nicaraguensis $\mathrm{BC}_{2} \mathrm{~F}_{1}$ population for the Qaer1.06 region of chromosome 1 by using the 36 SSR and INDEL markers together with the previous mapped 6 SSR markers. The target region of the Qaer1.06, from bnlg1832 (bin $1.05)-\mathrm{umc} 1128$ (1.07) covered $46.9 \mathrm{cM}$ at an average interval of $1.1 \mathrm{cM}$ per marker (Fig. 4B). The map distance of 46.9 $\mathrm{cM}$ was very close to that using only the 6 SSR markers of the same mapping population at $46.5 \mathrm{cM}$ (Fig. 4C, Mano and Omori 2008). These results suggested the absence of double recombination and the genotyping of the markers was accurate.

In the $\mathrm{BC}_{4} \mathrm{~F}_{1}$ population, we also constructed a linkage map using the newly identified 36 markers and previous mapped 6 SSRs. The order of markers corresponded well across the two maps. The map distance of $17.6 \mathrm{cM}$ from bnlg1832-umc1128 was shorter (37.5\%) and the recombination was strongly suppressed compared to the $\mathrm{BC}_{2} \mathrm{~F}_{1}$ map of $46.9 \mathrm{cM}$ (Fig. 4A and Fig. 4B). The suppression was also found in the region for bin 1.07 to $1.12\left(50.4 \mathrm{cM}\right.$ in $\mathrm{BC}_{2} \mathrm{~F}_{1}$ vs. $27.1 \mathrm{cM}$ in $\mathrm{BC}_{4} \mathrm{~F}_{1}$; data not shown).

\section{Mapping QTL for aerenchyma formation in the $B C_{4} F_{1}$ pop-} ulation

Through CIM analysis, a QTL for aerenchyma formation was identified at umc1297-IDP5918 (bin 1.05, LOD $=8.2$, effect $=0.25$ ), which explained $25 \%$ of the total phenotypic variance (Table 1). Z. nicaraguensis, with high degree of aerenchyma, contributed to the QTL. Distance of LOD peak 
Table 1. Chromosome locations and mode of gene action of QTLs for root aerenchyma formation estimated by composite interval mapping $(\mathrm{CIM})$ in the $\mathrm{BC}_{4} \mathrm{~F}_{1}$ population of the cross between $\mathrm{Mi} 29 \times$ Z. nicaraguensis

\begin{tabular}{|c|c|c|c|c|c|c|c|c|}
\hline QTL & Chr & Bin & Position $^{a}$ & Marker interval & $\mathrm{LOD}^{c}$ & Effect $^{d}$ & $\operatorname{Dir}^{e}$ & $\operatorname{Var}^{f}$ \\
\hline Qaer1.05-6 & 1 & 1.05 & $45(36-47)^{b}$ & umc1297-IDP5918 & 8.2 & 0.25 & $\mathrm{~N}$ & 0.25 \\
\hline Qaer8.05 & 8 & 8.05 & $0(0-12)$ & umc1712-bnlg162 & 2.6 & 0.17 & $\begin{array}{c}\mathrm{N} \\
\text { Total }\end{array}$ & $\begin{array}{l}0.07 \\
0.31\end{array}$ \\
\hline
\end{tabular}

${ }^{a}$ QTL position in cM from the top of the segregating markers. bnlg1484 (bin 1.03) for chromosome 1 and umc1712 (8.05) for chromosome 8.

b 2-LOD support interval.

${ }^{c}$ Significant thresholds level $(\mathrm{LOD}=2.0)$ was determined by 1,000 permutation test.

${ }^{d}$ Heterozygous (Mi29/Z. nicaraguensis)-homozygous for Mi29 alleles.

${ }^{e}$ Parent contributing higher-value allele, where $\mathrm{N}=Z$. nicaraguensis.

$f$ Proportion of phenotyphic variance explained.

between the $\mathrm{BC}_{2} \mathrm{~F}_{1}$ (bin 1.06, Mano and Omori 2008) and $\mathrm{BC}_{4} \mathrm{~F}_{1}$ analyses (bin 1.05 , this study) was about $20 \mathrm{cM}$ based on the map distance of the $\mathrm{BC}_{2} \mathrm{~F}_{1}$ population and about $8 \mathrm{cM}$ based on that of the $\mathrm{BC}_{4} \mathrm{~F}_{1}$ population, respectively (Fig. 4). We hereby designate the QTL as "Qaer1.05-6". In addition to chromosome 1 , small regions on chromosomes 3, 4, 7 and 8 were segregated in the $\mathrm{BC}_{4} \mathrm{~F}_{1}$ population (Fig. 1). QTL analysis in these regions identified a minor and new QTL at the interval of umc1712-bnlg162 on chromosome 8 (bin 8.05, $\left.\mathrm{LOD}=2.6, \mathrm{r}^{2}=0.07\right)$ (Table 1).

\section{Discussion}

In 1989, a new species of teosinte $Z$. nicaraguensis was discovered in far northwest Nicaragua in an area that is frequently flooded for many weeks (Bird 2000, Iltis and Benz 2000). Using the unique germplasm $Z$. nicaraguensis, we have analyzed several flooding tolerance-related traits including the capacity to form constitutive aerenchyma and the ability to grow flooding-induced adventitious roots at the soil surface (Mano and Omori 2007, Kindiger and Mano 2009). In this study, using the $\mathrm{BC}_{2} \mathrm{~F}_{1}$ and $\mathrm{BC}_{4} \mathrm{~F}_{1}$ populations from the $\mathrm{Mi} 29 \times Z$. nicaraguensis cross, we constructed a high-density linkage map bracketing the region at the aerenchyma locus Qaer1.05-6. In addition, we have confirmed a positive effect of the Qaer1.05-6 on aerenchyma formation in the genetic background of individuals of a genotype that is approximately $80 \%$ Mi29 (Fig. 1). The high-density linkage map and $\mathrm{BC}_{4} \mathrm{~F}_{1}$ segregants developed in this study can allow the detailed mapping of the Qaer1.05-6 and the generation of near-isogenic lines possessing an extremely small chromosome segment of $Z$. nicaraguensis.

The map length around the Qaer1.05-6 in the evaluated Mi29 $\times Z$. nicaraguensis $\mathrm{BC}_{4} \mathrm{~F}_{1}$ population of $17.6 \mathrm{cM}$ was much shorter $(37.5 \%)$ than $\mathrm{BC}_{2} \mathrm{~F}_{1}$ population map (Fig. 4), suggesting the presence of recombination suppression in the $\mathrm{BC}_{4} \mathrm{~F}_{1}$ population. This observation was also confirmed in the next generation of $\mathrm{BC}_{5} \mathrm{~F}_{1}$ map of $19.1 \mathrm{cM}$ at the same interval (Y. Mano, unpublished). The recombination suppression in the $\mathrm{BC}_{4} \mathrm{~F}_{1}$ generation was chromosome 1 specific because the suppression was not observed (rather extended) at the remaining regions on chromosomes 3,4 and 8 in the $\mathrm{BC}_{4} \mathrm{~F}_{1}$ map (data not shown). In an earlier study utilizing interspecies crosses in tomato, recombination between pairs of markers was less in the $\mathrm{BC}_{2} \mathrm{~F}_{2}$ (developed from heterozygous chromosome segments flanked by homozygous regions) than in the $\mathrm{BC}_{1}$ generation (developed from intact chromosomes) (Paterson et al. 1988, 1990). The authors suggested that chiasmata formation may be more frequent in homozygous regions, at the expense of recombination in heterozygous segments. In our study, above explanation may not be generally applicable since the chromosome segment of $Z$. nicaraguensis is relatively large on chromosome 1 in the $\mathrm{BC}_{3} \mathrm{~F}_{1}$ plant (Fig. 1).

Using the $\mathrm{BC}_{2} \mathrm{~F}_{1}$ population from $\mathrm{Mi} 29 \times$ Z. nicaraguensis cross, the peak of the QTL was located at the bin 1.06 (Mano and Omori 2008). In this study using the $\mathrm{BC}_{4} \mathrm{~F}_{1}$ population of the same cross combination, the position of the QTL was shifted to the direction of the short arm compared to the $\mathrm{BC}_{2} \mathrm{~F}_{1}$ population and the distance between the LOD peak is $8-20 \mathrm{cM}$. Because we developed the 2 populations from the same single $\mathrm{F}_{1}$ plant, the difference of the QTL positions between the $\mathrm{BC}_{2} \mathrm{~F}_{1}$ and $\mathrm{BC}_{4} \mathrm{~F}_{1}$ populations is not due to the fact that the $Z$. nicaraguensis is not a pure line or different genetic composition. The difference is also not due to different marker density because when the QTL mapping in the $\mathrm{BC}_{2} \mathrm{~F}_{1}$ population was re-calculated using the highdensity linkage map developed in this study, the same peak was observed (data not shown). We will soon identify this QTL position more precisely using the developmental lines possessing a series of chromosome segments at the bin 1.057 region together with high-density linkage map.

Other than Qaer1.05-6, several QTLs controlling root aerenchyma formation with minor effects have been found in the teosintes. In maize $\times Z$. nicaraguensis mapping populations, QTLs other than Qaer1.05-6 were located on chromosome 1 (Qaer1.01, Qaer1.02-3, Qaer1.11), chromosome 5 (Qaer5.01, Qaer5.09, Qaer5.09n) and chromosome 8 (Qaer8.06-7) (Mano et al. 2007, Mano and Omori 2008). In this study, minor QTL was identified on chromosome 8 at bin 8.05 (Qaer8.05). The mode of gene action of the Qaer8.06-7 was overdominance with negative effects (Mano et al. 2007), whereas that in the Qaer8.05 was positive action when in the heterozygous genotype (Table 1). This suggests the two are different loci and the Qaer8.05 is a newly identified QTL. As well as the ability to grow 
flooding-induced adventitious roots at the soil surface (Mano et al. 2005), the capacity to form root aerenchyma is complex trait and is controlled by multiple genes. Nevertheless, we have acquired much QTL information regarding aerenchyma formation and it may now be possible to transfer and pyramid the QTLs from teosinte into maize via marker-assisted selection.

Inducible aerenchyma formation in plant roots is promoted by accumulation of endogenous ethylene (summarized by Evans 2003, Shiono et al. 2008) and many ethylene or hypoxia-induced genes have been reported (Saab and Sachs 1996). Recently, Mühlenbock et al. (2007) reported, in Arabidopsis hypocotyls, that the balanced activities of LESION SIMULATING DISEASE1 (LSD1), ENHANCED DISEASE SUSCEPTIBILITY1 (EDS1) and PHYTOALEXIN DEFICIENT4 (PAD4) regulate lysigenous aerenchyma formation in response to hypoxia. However, gene isolation with regard to inducible and/or constitutive root aerenchyma forming process has not been reported in crops because lack of suitable experimental materials (e.g. mutant and isogenic line). We will soon have available near isogenic lines of Mi29 possessing QTL for aerenchyma formation with only an extremely small chromosome segment of $Z$. nicaraguensis.

Laser microdissection has been used to analyze specific cells of interest (e.g. cortical tissue) from heterogeneous tissues (Nakazono et al. 2003, Ohtsu et al. 2007b). In addition, Margulies et al. (2005) reported a high parallel sequencing system (454 sequencing) that achieves 100-fold increase in throughput compared to current sequencing methods, and it has been applied for SNP discovery (Barbazuk et al. 2007) and global gene expression analysis in maize (Ohtsu et al. 2007a). In addition to map-based cloning, it will be possible to isolate constitutive aerenchyma-forming gene(s) from cortical tissue in roots of the developed isogenic line by applying a combination of laser microdissection and 454 transcriptome sequencing analysis.

\section{Acknowledgments}

The authors wish to thank the International Maize and Wheat Improvement Center (CIMMYT), Mexico for providing seed of Z. nicaraguensis and the National Agricultural Research Center for Kyushu Okinawa Region for supplying maize Mi29. They also thank Dr. B. Kindiger (USDA/ ARS), Dr. M. Nakazono (The University of Tokyo), Dr. A. Oyanagi (NICS) and Dr. K. Kawaguchi (NICS) for critically reviewing the manuscript and Dr. H. Takahashi (Akita Prefectural University), Dr. R. McK. Bird (North Carolina State University) and Dr. C.H. Loaisiga (Universidad Nacional Agraria) for contributing to the work. The research was supported by a grant from the Bio-oriented Technology Research Advancement Institution (Promotion of Basic Research Activities for Innovative Biosciences, No. H20/ seeds-01-02) and by the National Agriculture and Food Research Organization (NARO), Japan.

\section{Literature Cited}

Barbazuk, W.B., S.J.Emrich, H.D.Chen, L.Li and P.S.Schnable (2007) SNP discovery via 454 transcriptome sequencing. Plant J. 51: 910 918.

Bird, R.McK. (2000) A remarkable new teosinte from Nicaragua: Growth and treatment of progeny. Maize Gen. Coop. Newsl. 74: $58-59$.

Boru, G., M. van Ginkel， R.M. Trethowan, L. Boersma and W.E. Kronstad (2003) Oxygen use from solution by wheat genotypes differing in tolerance to waterlogging. Euphytica 132: 151-158.

Churchill,G.A. and R.W.Doerge (1994) Empirical threshold values for quantitative trait mapping. Genetics 138: 963-971.

Colmer,T.D. (2003) Aerenchyma and an inducible barrier to radial oxygen loss facilitate root aeration in upland, paddy and deep-water rice (Oryza sativa L.). Ann. Bot. 91: 301-309.

Colmer,T.D. and L.A.C.J.Voesenek (2009) Flooding tolerance: suites of plant traits in variable environments. Funct. Plant Biol. 36: 665681.

Doebley,J.F. and H.H.Iltis (1980) Taxonomy of Zea (Gramineae). I. A subgeneric classification with key to taxa. Am. J. Bot. 67: 982993

Evans,D.E. (2003) Aerenchyma formation. New Phytol. 161: 35-49.

Gunawardena, A.H.L.A.N., D.M. Pearce, M.B.Jackson, C.R. Hawes and D.E.Evans (2001) Characterisation of programmed cell death during aerenchyma formation induced by ethylene or hypoxia in roots of maize (Zea mays L.). Planta 212: 205-214.

He, C.J., S.A.Finlayson, M.C.Drew, W.R.Jordan and P.W.Morgan (1996) Ethylene biosynthesis during aerenchyma formation in roots of maize subjected to mechanical impedance and hypoxia. Plant Physiol. 112: 1679-1685.

Hochholdinger,F. and R.Tuberosa (2009) Genetic and genomic dissection of maize root development and architecture. Curr. Opin. Plant Biol. 12: 172-177.

Huang,B., J.W.Johnson, D.S.NeSmith and D.C.Bridges (1994) Root and shoot growth of wheat genotypes in response to hypoxia and subsequent resumption of aeration. Crop Sci. 34: 1538-1544.

Iltis,H.H. and B.F.Benz (2000) Zea nicaraguensis (Poaceae), a new teosinte from Pacific coastal Nicaragua. Novon 10: 382-390.

Iltis,H.H. and J.F.Doebley (1980) Taxonomy of Zea (Gramineae). II Subspecific categories in the Zea mays complex and a generic synopsis. Am. J. Bot. 67: 994-1004.

Jackson,M.B., T.M.Fenning, M.C.Drew and L.R.Saker (1985a) Stimulation of ethylene production and gas-space (aerenchyma) formation in adventitious roots of Zea mays L. by small partial pressure of oxygen. Planta 165: 486-492.

Jackson, M.B., T.M.Fenning and W.Jenkins (1985b) Aerenchyma (gas-space) formation in adventitious roots of rice (Oryza sativa L.) is not controlled by ethylene or small partial pressures of oxygen. J. Exp. Bot. 36: 1566-1572.

Justin, S.H.F.W. and W.Armstrong (1987) The anatomical characteristics of roots and plant response to soil flooding. New Phytol. 106: 465-495.

Kindiger,B. and Y.Mano (2009) Maize root characteristics that enhance flooding tolerance. In: Proc Joint Annual Meetings of the American Society of Plant Biologists and the Phycological Society of America, 18-22 July 2009, Honolulu, Hawaii, pp. 372-373.

Komatsuda,T., I.Nakamura, F.Takaiwa and S.Oka (1998) Development of STS markers closely linked to the vrs1 locus in barley, Hordeum vulgare. Genome 41: 680-685.

Lander,E.S., P.Green, J.Abrahamson, A. Barlow, M.J.Daly, S.E. 
Lincoln and L.Newburg (1987) MAPMAKER: An interactive computer package for constructing primary genetic linkage maps of experimental and natural populations. Genomics 1: 174-181.

Mano, Y., M.Muraki, M.Fujimori, T.Takamizo and B. Kindiger (2005) Identification of QTL controlling adventitious root formation during flooding conditions in teosinte (Zea mays ssp. huehuetenangensis) seedlings. Euphytica 142: 33-42.

Mano, Y., F. Omori, T. Takamizo, B. Kindiger, R.McK. Bird and C.H.Loaisiga (2006) Variation for root aerenchyma formation in flooded and non-flooded maize and teosinte seedlings. Plant Soil 281: 269-279.

Mano,Y. and F.Omori (2007) Breeding for flooding tolerant maize using "teosinte" as a germplasm resource. Plant Root 1: 17-21.

Mano, Y., F. Omori, T.Takamizo, B.Kindiger, R.McK. Bird, C.H. Loaisiga and H.Takahashi (2007) QTL mapping of root aerenchyma formation in seedlings of a maize $\times$ rare teosinte "Zea nicaraguensis" cross. Plant Soil 295: 103-113.

Mano,Y. and F.Omori (2008) Verification of QTL controlling root aerenchyma formation in a maize $\times$ teosinte "Zea nicaraguensis" advanced backcross population. Breed. Sci. 58: 217-223.

Mano,Y., F.Omori, B.Kindiger and H.Takahashi (2008) A linkage map of maize $\times$ teosinte Zea luxurians and identification of QTLs controlling root aerenchyma formation. Mol. Breed. 21:327-337.

Margulies, M., M.Egholm, W.E.Altman, S.Attiya, J.S.Bader, L.A. Bemben, J.Berka, M.S.Braverman, Y.-J.Chen, Z.Chen et al. (2005) Genome sequencing in microfabricated high-density picolitre reactors. Nature 437: 376-380.

Mühlenbock, P., M.Plaszczyca, M.Plaszczyca, E.Mellerowicz and S.Karpinski (2007) Lysigenous aerenchyma formation in Arabidopsis is controlled by LESION SIMULATING DISEASE1. Plant Cell 19: 3819-3830.

Nakazono, M., F.Qiu, L.A.Borsuk and P.S.Schnable (2003) Lasercapture microdissection, a tool for the global analysis of gene expression in specific plant cell types: Identification of genes expressed differentially in epidermal cells or vascular tissues of maize. Plant Cell 15: 583-596.

Nelson,J.C. (1997) QGENE: Software for marker-based genomic analysis and breeding. Mol. Breed. 3: 239-245.

Ohtsu, K., M.B.Smith, S.J.Emrich，L.A.Borsuk，R.Zhou，T.Chen, X.Zhang, M. Timmermans, J.Beck, B.Buckner et al. (2007a) Global gene expression analysis of the shoot apical meristem of maize (Zea mays L.). Plant J. 52: 391-404.

Ohtsu,K., H.Takahashi, P.S.Schnable and M.Nakazono (2007b) Cell type-specific gene expression profiling in plants by using a combination of laser microdissection and high-throughput technologies. Plant Cell Physiol. 48: 3-7.
Pang, J., M.Zhou, N.Mendham and S.Shabala (2004) Growth and physiological responses of six barley genotypes to waterlogging and subsequent recovery. Aust. J. Agric. Res. 55: 895-906.

Paterson,A.H., E.S.Lander, J.D.Hewitt, S.Peterson, S.E.Lincoln and S.D.Tanksley (1988) Resolution of quantitative traits into Mendelian factors by using a complete linkage map of restriction fragment length polymorphism. Nature 335: 721-726.

Paterson,A.H., J.W.DeVerna, B.Lanini and S.D.Tanksley (1990) Fine mapping of quantitative trait loci using selected overlapping recombinant chromosomes, in an interspecies cross of tomato. Genetics 124: 735-742.

Ray,J.D., B.Kindiger and T.R.Sinclair (1999) Introgressing root aerenchyma into maize. Maydica 44: 113-117.

Saab, I.N. and M.M.Sachs (1996) A flooding-induced xyloglucan endo-transglycosylase homolog in maize is responsive to ethylene and associated with aerenchyma. Plant Physiol. 112: 385-391.

Setter,T.L. and I.Waters (2003) Review of prospects for germplasm improvement for waterlogging tolerance in wheat, barley and oats. Plant Soil 253: 1-34.

Shiono,K., H.Takahashi, T.D.Colmer and M.Nakazono (2008) Role of ethylene in acclimations to promote oxygen transport in roots of plants in waterlogged soils. Plant Sci. 175: 52-58.

Smirnoff,N. and R.M.M.Crawford (1983) Variation in the structure and response to flooding of root aerenchyma in some wetland plants. Ann. Bot. 51: 237-249.

Stanca, A.M., I. Romagosa, K. Takeda, T.Lundborg, V.Terzi and L.Cattivelli (2003) Diversity in abiotic stress tolerances. In: Bothmer,R.v., T.v.Hintum, H.Knüpffer and K.Sato (eds.) Diversity in Barley (Hordeum vulgare), Elsevier, Amsterdam, p. 280.

Subbaiah,C.C. and M.M.Sachs (2003) Molecular and cellular adaptations of maize to flooding stress. Ann. Bot. 91: 119-127.

Takeda,K. (1989) Varietal variation of flooding tolerance in barley seedlings, and its diallel analysis. Jpn J. Breed. 39 (Suppl. 1): 174175.

Wang, S., C.J.Basten and Z.-B.Zeng (2006) Windows QTL Cartographer 2.5. Department of Statistics, North Carolina State University, Raleigh, NC, USA. http://statgen.ncsu.edu/qtlcart/WQTLCart.htm

Watkin,E.L.J., C.J.Thomson and H.Greenway (1998) Root development and aerenchyma formation in two wheat cultivars and one triticale cultivar grown in stagnant agar and aerated nutrient solution. Ann. Bot. 81: 349-354.

Zaidi,P.H., P.Maniselvan, P.Yadav, A.K.Singh, R.Sultana, P.Dureja, R.P.Singh and G. Srinivasan (2007) Stress-adaptive changes in tropical maize (Zea mays L.) under excessive soil moisture stress. Maydica 52: 159-171. 